Pacific Journal of Mathematics

A NEW CLASS OF ISOCOMPACT SPACES AND RELATED Masai Sara 


\title{
A NEW CLASS OF ISOCOMPACT SPACES AND RELATED RESULTS
}

\section{MaSAmi SaKaI}

\begin{abstract}
In the last fifteen years a large number of classes of isocompact spaces were investigated by many mathematicians. In this paper we introduce a new large class (i.e. the class of $k$-neat spaces) of isocompact spaces. This class contains all of the following classes: neighborhood $\mathscr{F}$-spaces, spaces satisfying property $\theta L$, weakly $\left[\omega_{1}, \infty\right)^{r}$-refinable spaces, $\delta \theta$-penetrable spaces and pure spaces. Other properties of this class are also investigated. For example we show that an $\omega_{1}$-compact $\omega_{1}$-neat $T_{1}$-space is $a$-realcompact and $k$-neatness is an inverse invariant of maps under some conditions. In the last section we consider compactness of isocompact spaces having a countably compact dense subset.
\end{abstract}

1. Introduction and preliminaries. A space is said to be isocompact if every closed countably compact subset is compact [2]. Since Bacon's paper [2], many isocompact classes have been found. In this paper we introduce a new large class (i.e. the class of $k$-neat spaces) of isocompact spaces. This class properly contains the class of neighborhood $\mathscr{F}_{\text {-spaces }}$ [7], spaces satisfying property $\theta L[6]$, weakly $\left[\omega_{1}, \infty\right)^{r}$-refinable spaces [21], $\delta \theta$-penetrable spaces [4], and pure spaces [1]. By this concept we can neatly review many results in the area of isocompactness.

In the second section the definition of $k$-neat spaces is given for an infinite cardinal $k$. It is proved that every $k$-neat space is isocompact and every $\omega_{1}$-compact $\omega_{1}$-neat $T_{1}$-space is $a$-realcompact (i.e. closed complete). These two theorems strengthen many results in this area. Behavior of $k$-neat spaces under some operations is investigated in the third section, and we give an example which demonstrates that neat spaces are strictly weaker than the isocompact classes listed above. In the fourth section we consider isocompact spaces having a countably compact dense subset, and provide conditions for such a space to be compact. As one case we show that a regular $T_{2}$ isocompact space is compact if it is represented as the union of a countably compact dense subset and an almost realcompact dense subset.

In this paper all maps are assumed to be continuous.

The rest of this section is devoted to some definitions used in the following sections. We denote by $\omega\left(\omega_{1}\right)$ the first infinite (uncountable) cardinal and $\mathscr{P}(X)$ denotes the power set of a set $X$. 
DeFINITION 1.1. [7] [13] A space $X$ is called an $\mathscr{F}$-space if there is a function $B: \omega \times X \rightarrow \mathscr{P}(X)$ such that the following are true:

(1) For each $n \in \omega$ and $x \in X, B(n+1, x) \subset B(n, x)$, and for each $x \in X, \cap_{n \in \omega} B(n, x)=\{x\}$.

(2) A subset $U \subset X$ is open if and only if, for each $x \in U$, there exists $n_{x} \in \omega$ such that $B\left(n_{x}, x\right) \subset U$.

(3) If $F \subset X$ is closed and $x \notin F$, then there exists $n \in \omega$ such that for each $y \in B(n, x)-\{x\}$, there exists $n_{y} \in \omega$ such that $\{x, y\} \not \subset$ $\cup_{f \in F} B\left(n_{y}, f\right)$.

We say $X$ is a neighborhood $\mathscr{F}$-space if $B(n, x)$ is an open neighborhood of $x$ for each $n \in \omega$ and $x \in X$.

We denote by Card the class of all infinite cardinals. For a collection $\mathscr{V}$ of subsets of a set, $\omega \mathscr{V}$ is the set of unions of countable subcollections of $\mathscr{V}$.

Definition 1.2. [6] For $k \in$ Card, and $\mathscr{U}$ and $\mathscr{V}$ collections of subsets of a space $X$, we say $\mathscr{V}$ is $k$-weakly cushioned in $\mathscr{U}$ if and only if there exists a function $f$ from $\mathscr{V}$ to $\mathscr{U}$ such that if $\mathscr{W} \subset \mathscr{V}$ with $|\mathscr{W}| \leq k$ and $x: \mathscr{W} \rightarrow \cup \mathscr{W}$ with $x(G) \in G$ for each $G \in \mathscr{W}$, then $\overline{\{x(G): G \in \mathscr{W}\}} \subset \cup f(\mathscr{W})$.

Definition 1.3. [6] For $k \in$ Card, we say a space $X$ satisfies property $\theta k L$ if and only if for every open cover $\mathscr{U}$ of $X$ there exists a sequence $\left\langle\mathscr{D}_{n}: n \in \omega\right\rangle$ of collections of subsets of $X$ and a sequence $\left\langle\mathscr{V}_{n}: n \in \omega\right\rangle$ of open refinements of $\mathscr{U}$ such that $\cup_{n \in \omega} \mathscr{D}_{n}$ covers $X$ and for each $n \in \omega, \cup \mathscr{D}_{n} \subset \cup \mathscr{V}_{n}$ and $\mathscr{D}_{n}$ is $k$-weakly cushioned in $\omega \mathscr{V}_{n}$ in the space $\cup \mathscr{V}_{n}$.

We shall refer to property $\theta \omega L$ as property $\theta L$.

Spaces satisfying property $L[2]$ and weakly $\delta \theta$-refinable spaces [19] satisfy property $\theta L[6$, Theorem $2.2,2.3]$. For $\mathscr{B}$ a collection of subsets of a space $X$ and $x \in X$, set $I(x, \mathscr{B})=\bigcap\{B: B \in \mathscr{B}, x \in B\}$ and $\operatorname{ord}(x, \mathscr{B})$ denotes the cardinality of the set $\{B: B \in \mathscr{B}, x \in B\}$.

Definition 1.4. [4] We say that an open cover $\bigcup_{n \in \omega} \mathscr{V}_{n}$ of a space $X$ is a $\theta$-penetration (resp. $\delta \theta$-penetration) of a cover $\mathscr{U}$ of $X$ if, for every $x \in X, \cap\left\{I\left(x, \mathscr{V}_{n}\right): n \in \omega\right.$ and $\left.0<\operatorname{ord}\left(x, \mathscr{V}_{n}\right)<\omega\right\} \subset U$ for some $U \in \mathscr{U}$ (resp. $\bigcap\left\{\left(x, \mathscr{V}_{n}\right): n \in \omega\right.$ and $\left.0<\operatorname{ord}\left(x, \mathscr{V}_{n}\right) \leq \omega\right\} \subset U$ for some $U \in \mathscr{U}$ ), and that $X$ is $\theta$-penetrable (resp. $\delta \theta$-penetrable) if every open cover of $X$ has a $\theta$-penetration (resp. $\delta \theta$-penetration). 
Spaces with a point countable separating open cover and weakly $\delta \theta$-refinable spaces are $\delta \theta$-penetrable [4, Remarks 2.1].

Definition 1.5. [21] A space $X$ is said to be weakly $\left[\omega_{1}, \infty\right)^{r}$-refinable if for any open cover $\mathscr{U}$ of uncountable regular cardinality there exists an open refinement which can be expressed as $\bigcup_{\gamma \in \Gamma} \mathscr{V}_{\gamma}$, where $|\Gamma|<|\mathscr{U}|$ and if $x \in X$ there is some $\gamma \in \Gamma$ such that $0<\operatorname{ord}\left(x, \mathscr{V}_{\gamma}\right)<|\mathscr{U}|$.

Obviously weakly $\delta \theta$-refinable spaces are weakly $\left[\omega_{1}, \infty\right)^{r}$-refinable.

A cover $\mathscr{E}=\bigcup_{n \in \omega} \mathscr{E}_{n}$ of a space $X$ is called an interlacing if for each $n \in \omega$ and $U \in \mathscr{E}_{n}, U$ is open in $\mathscr{E}_{n}^{*}$, where $\mathscr{E}_{n}^{*}=\bigcup\left\{E: E \in \mathscr{E}_{n}\right\}$. Let $\mathscr{H}$ be a family of subsets of $X$. We say that an interlacing $\mathscr{E}=\bigcup \mathscr{E}_{n}$ is $\delta$-suspended from $\mathscr{H}$ if for each $n \in \omega$ and $x \in \mathscr{E}_{n}^{*}$ there exists a countable subfamily $\mathscr{F}$ of $\mathscr{H}$ such that $\operatorname{St}\left(x, \mathscr{E}_{n}\right) \cap(\cap \mathscr{F})=\varnothing$.

Definition 1.6. [1] A space $X$ is said to be pure (ultrapure) if for each free closed ultrafilter(filter) $\mathscr{H}$ on $X$ with countable intersection property (c.i.p.) there exists an interlacing on $X$ that is $\delta$-suspended from $\mathscr{H}$.

Ultrapure and astral spaces [1], spaces with a quasi- $G_{\delta}$-diagonal [15], almost realcompact spaces [10], weakly Borel complete spaces [17] and $a$-realcompact spaces [9] are all pure.

2. $k$-neat spaces. Define for each free closed ultrafilter $\mathscr{H}$ on $X$ with c.i.p., $\lambda(\mathscr{H})=\min \{|\mathscr{F}|: \mathscr{F} \subset \mathscr{H}, \cap \mathscr{F}=\varnothing\} . \lambda(\mathscr{H})$ is an uncountable regular cardinal.

Definition 2.1. Let $\mathscr{H}$ be a free closed ultrafilter on $X$ with c.i.p. and $k \in$ Card. A system $\left\langle\left\{X_{\gamma}\right\},\left\{\mathscr{V}_{\gamma}\right\},\left\{f_{\gamma}\right\}\right\rangle_{\gamma \in \Gamma}$ is called a $k$-neat system for $\mathscr{H}$ if the following are satisfied:

(1) $|\Gamma|<\lambda(\mathscr{H})$.

(2) $\left\{X_{\gamma}\right\}_{\gamma \in \Gamma}$ is a cover of $X$ and $\mathscr{V}_{\gamma}$ is an open collection of $X$ such that $X_{\gamma} \subset \mathscr{V}_{\gamma}^{*}$ for each $\gamma \in \Gamma$.

(3) Each $f_{\gamma}$ is a function from $X_{\gamma}$ to $\mathscr{V}_{\gamma}$ such that if $A \subset X_{\gamma},|A| \leq k$ and $f_{\gamma} \mid A$ is injective, then the closure of $A$ in $\mathscr{V}_{\gamma}{ }^{*}$ is contained in $\bigcup_{x \in A} f_{\gamma}(x)$.

(4) For each $\gamma \in \Gamma$ and $x \in X_{\gamma}$ there exists $H \in \mathscr{H}$ such that $f_{\gamma}(x) \cap X_{\gamma} \cap H=\varnothing$. 
A space $X$ is called a $k$-neat space if for each free closed ultrafilter $\mathscr{H}$ on $X$ with c.i.p. there exists a $k$-neat system for $\mathscr{H}$. We shall refer to an $\omega$-neat space as merely a neat space.

A $k^{\prime}$-neat space is $k$-neat if $k^{\prime} \geq k$. It is easily seen that for all $k \in$ Card, a space $X$ with countable tightness is $k$-neat if and only if $X$ is neat.

LeMMa 2.2. [4, Lemma 2.2] If $\mathscr{H}$ is a free closed ultrafilter on $X$ with c.i.p. and if $\mathscr{V}=\bigcup_{n \in \omega} \mathscr{V}_{n}$ is a $\theta$-penetration (resp. $\delta \theta$-penetration) of $\mathscr{U}=\{X-H: H \in \mathscr{H}\}$, then $\mathscr{V}$ has a subcover that is a weak $\theta$-refinement (resp. weak $\delta \theta$-refinement) of $\mathscr{U}$.

Proposition 2.3. The following spaces are neat. Moreover, the implications $(\mathrm{a}) \rightarrow(\mathrm{b})$ and $(\mathrm{d}) \rightarrow(\mathrm{e})$ hold.

(a) neighborhood $\mathscr{F}$-spaces.

(b) spaces satisfying property $\theta L$.

(c) weakly $\left[\omega_{1}, \infty\right)^{r}$-refinable spaces.

(d) $\delta \theta$-penetrable spaces.

(e) pure spaces.

Proof. (a) $\rightarrow$ (b) Let $\mathscr{U}$ be any open cover of $X$, and set $S=\{x \in X$ : $x \in \cup_{Z \in X-U} B(n, z)$ for each $n \in \omega$ and $\left.x \in U \in \mathscr{U}\right\}$. We note that $S$ is a discrete subset of $X$. Take $x \in S$, and select $U_{x} \in \mathscr{U}$ such that $x \in U_{x}$. For $x$ and $X-U_{x}$ there exists $n_{x} \in \omega$ corresponding to (3) of Definition 1.1. We may assume $B\left(n_{x}, x\right) \subset U_{x}$. Since for $y \in B\left(n_{x}, x\right)-$ $\{x\}$ there exists $n_{y} \in \omega$ such that $\{x, y\} \not \subset U_{Z \in X-U_{x}} B\left(n_{y}, z\right)$, it follows from $x \in \cup_{Z \in X-U_{x}} B\left(n_{y}, z\right)$ that $y \notin \bigcup_{Z \in X-U_{x}} B\left(n_{y}, z\right)$. So $y \notin S$ for any $y \in B\left(n_{x}, x\right)-\{x\}$. Thus $S$ is discrete in $X$. Set $\mathscr{D}_{0}=\{\{x\}$ : $x \in S\}, \mathscr{V}_{0}=\left\{B\left(n_{x}, x\right): x \in S\right\}$ and define a function $f_{0}$ from $\mathscr{D}_{0}$ to $\mathscr{V}_{0}$ such that $f_{0}(\{x\})=B\left(n_{x}, x\right) . \mathscr{D}_{0}$ is $k$-weakly cushioned in $\mathscr{V}_{0}$ in the space $\mathscr{V}_{0}^{*}$ for any $k \in$ Card.

For $x \in X-S$ we can take $U_{x} \in \mathscr{U}$ and $n_{x} \in \omega-\{0\}$ such that $x \in U_{x}$ and $x \notin \bigcup_{Z \in X-U_{x}} B\left(n_{x}, z\right)$. Put $X_{n}=\left\{x \in X-S: n_{x}=n\right\}$. Obviously $X-S=\bigcup_{n=1}^{\infty} X_{n}$. Set $\mathscr{D}_{n}=\left\{\{x\}: x \in X_{n}\right\}, \mathscr{V}_{n}=\left\{U_{x}: x \in\right.$ $\left.X_{n}\right\}$ and define a function $f_{n}$ from $\mathscr{D}_{n}$ to $\mathscr{V}_{n}$ such that $f_{n}(\{x\})=U_{x}$ for $n \geq 1$. It is easily proved that $\mathscr{D}_{n}$ is $k$-weakly cushioned in $\mathscr{V}_{n}$ in the space $\mathscr{V}_{n}{ }^{*}$ for $k \in$ Card. Thus $X$ satisfies property $\theta k L$.

(b) Let $\mathscr{H}$ be a free closed ultrafilter on $X$ with c.i.p.. Then 
$\mathscr{U}=\{X-H: H \in \mathscr{H}\}$ is an open cover of $X$. For this $\mathscr{U}$ there exist sequences $\left\langle\mathscr{D}_{n}: n \in \omega\right\rangle$ and $\left\langle\mathscr{V}_{n}: n \in \omega\right\rangle$ of Definition 1.3. Let $f_{n}$ be a function of Definition 1.2 from $\mathscr{D}_{n}$ to $\omega \mathscr{V}_{n}$. We may assume that each $\mathscr{D}_{n}$ is a disjoint collection and each $f_{n}$ is injective. Put $X_{n}=\mathscr{D}_{n}^{*}$. For each $n \in \omega$ and $x \in X_{n}$ there exists uniquely $D_{x} \in \mathscr{D}_{n}$ such that $x \in D_{x}$. Put $W_{x}=f_{n}\left(D_{x}\right), \mathscr{W}_{n}=\left\{W_{x}: x \in X_{n}\right\}$ and define a function $g_{n}$ from $X_{n}$ to $\mathscr{W}_{n}$ such that $g_{n}(x)=W_{x} .\left\langle\left\{X_{n}\right\},\left\{\mathscr{W}_{n}\right\},\left\{g_{n}\right\}\right\rangle_{n \in \omega}$ is a desired neat system for $\mathscr{H}$.

(c) Let $\mathscr{H}$ be a free closed ultrafilter on $X$ with c.i.p. We take a free subfamily $\mathscr{F}$ of $\mathscr{H}$ such that $|\mathscr{F}|=\lambda(\mathscr{H})$. Since the cardinality of the open cover $\mathscr{U}=\{X-F: F \in \mathscr{F}\}$ is uncountable regular, there exists an open refinement $\mathscr{G}=\bigcup_{\gamma \in \Gamma} \mathscr{G}_{\gamma}$ of $\mathscr{U}$ such that $|\Gamma|<\lambda(\mathscr{H})$ and for each $x \in X$ there exists $\gamma \in \Gamma$ such that $0<\operatorname{ord}\left(x, \mathscr{G}_{\gamma}\right)<\lambda(\mathscr{H})$. Now for each $\gamma \in \Gamma$ we put $X_{\gamma}=\left\{x \in X: 0<\operatorname{ord}\left(x, \mathscr{G}_{\gamma}\right)<\lambda(\mathscr{H})\right\}, \quad \mathscr{V}_{\gamma}=$ $\left\{\operatorname{St}\left(x, \mathscr{G}_{\gamma}\right): x \in X_{\gamma}\right\}$ and define a function $f_{\gamma}$ from $X_{\gamma}$ to $\mathscr{V}_{\gamma}$ such that $f_{\gamma}(x)=\operatorname{St}\left(x, \mathscr{G}_{\gamma}\right) \cdot\left\langle\left\{X_{\gamma}\right\},\left\{\mathscr{V}_{\gamma}\right\},\left\{f_{\gamma}\right\}\right\rangle_{\gamma \in \Gamma}$ is a $k$-neat system for $\mathscr{H}$ for $k \in$ Card. Hence $X$ is $k$-neat for $k \in$ Card.

(d) $\rightarrow$ (e) Let $\mathscr{H}$ be a free closed ultrafilter on $X$ with c.i.p. By Lemma 2.2 the open cover $\{X-H: H \in \mathscr{H}\}$ has a weak $\delta \theta$-refinement $\mathscr{U}=\bigcup_{n \in \omega} \mathscr{U}_{n}$. Put $X_{n}=\left\{x \in X: 0<\operatorname{ord}\left(x, \mathscr{U}_{n}\right) \leq \omega\right\}$ for $n \in \omega$. If we set $\mathscr{E}_{n}=\left\{X_{n} \cap U: U \in \mathscr{U}_{n}\right\}$, then $\mathscr{E}_{n}=\bigcup_{n \in \omega} \mathscr{E}_{n}$ is obviously an interlacing on $X$ that is $\delta$-suspended from $\mathscr{H}$.

(e) Let $\mathscr{H}$ be a free closed ultrafilter on $X$ with c.i.p. Since $X$ is pure, we can obtain an interlacing $\mathscr{E}=\bigcup_{n \in \omega} \mathscr{E}_{n}$ on $X$ which is $\delta$-suspended from $\mathscr{H}$. For each $n \in \omega$ and $E \in \mathscr{E}_{n}$ we take an open set $U(E)$ of $X$ such that $U=U(E) \cap \mathscr{E}_{n}^{*}$. Now for each $n \in \omega$ put $X_{n}=\mathscr{E}_{n}^{*}, \mathscr{V}_{n}=$ $\left\{\operatorname{St}\left(x, \mathscr{F}_{n}\right): x \in X_{n}\right\}$, where $\mathscr{F}_{n}=\left\{U(E): E \in \mathscr{E}_{n}\right\}$, and define a function $f_{n}$ from $X_{n}$ to $\mathscr{V}_{n}$ such that $f_{n}(x)=\operatorname{St}\left(x, \mathscr{F}_{n}\right) .\left\langle\left\{X_{n}\right\},\left\{\mathscr{V}_{n}\right\},\left\{f_{n}\right\}\right\rangle_{n \in \omega}$ is a desired $k$-neat system for $\mathscr{H}$ for $k \in$ Card. Hence $X$ is $k$-neat for $k \in$ Card.

Davis asked in [7, Question 4.2] whether every (neighborhood) $\mathscr{F}$-space satisfies property $\theta L$. The above implication (a) $\rightarrow$ (b) affirmatively answers the question in the case of neighborhood $\mathscr{F}$-spaces. The following lemma is easy.

LEMMA 2.4. Let $Y$ be a closed subspace of a space $X$, and $\mathscr{F}$ be a free closed ultrafilter on $Y$ with c.i.p. Then $\mathscr{H}=\{H: H$ is closed in $X$ and $H \cap Y \in \mathscr{F}\}$ is a free closed ultrafilter on $X$ with c.i.p. and $\lambda(\mathscr{F})=\lambda(\mathscr{H})$ holds. 
LEMMA 2.5. Every closed subspace of a $k$-neat space is $k$-neat.

Proof. Let $Y$ be a closed subspace of a $k$-neat space $X$, and $\mathscr{F}$ be a free closed ultrafilter on $Y$ with c.i.p. By Lemma $2.4 \mathscr{H}=\{H: H$ is closed in $X$ and $H \cap Y \in \mathscr{F}$ \} is a free closed ultrafilter on $X$ with c.i.p. and $\lambda(\mathscr{F})=\lambda(\mathscr{H})$. We take a $k$-neat system for $\mathscr{H}$. We naturally restrict the system to $Y$. It is easily seen that the restricted system is a $k$-neat system for $\mathscr{F}$.

THEOREM 2.6. A neat space is isocompact.

Proof. By the above lemma we show that a countably compact neat space is compact. Suppose that there exists a countably compact noncompact neat space $X$. Since $X$ is not Lindelöf, $X$ has an open cover $\mathscr{U}$ which has no countable subcover. We take a closed ultrafilter $\mathscr{H}$ on $X$ containing $\{X-U: U \in \mathscr{U}\}$. Now $\mathscr{H}$ is a free closed ultrafilter on $X$ with c.i.p. There exists a neat system $\left\langle\left\{X_{\gamma}\right\},\left\{\mathscr{V}_{\gamma}\right\},\left\{f_{\gamma}\right\}\right\rangle_{\gamma \in \Gamma}$ for $\mathscr{H}$. By the fact $|\Gamma|<\lambda(\mathscr{H})$ we can get $\gamma_{0} \in \Gamma$ such that $X_{\gamma_{0}} \cap H \neq \varnothing$ for any $H \in \mathscr{H}$. We fix this $\gamma_{0}$. There exists $F \in \mathscr{H}$ such that $F \subset \mathscr{V}_{\gamma_{0}}^{*}$ because $X-\mathscr{V}_{\gamma_{0}}^{*} \notin \mathscr{H}$. By the way of the selecting of $\gamma_{0}$ we can obtain a countable subset $A=\left\{x_{n}: n \in \omega\right\}$ of $F \cap X_{\gamma_{0}}$ which satisfies $f_{\gamma_{0}}\left(x_{n}\right) \cap$ $\left\{x_{j}: j \geq n+1\right\}=\varnothing$ for any $n \in \omega$. Take an $\omega$-limit point $x$ of $A$ (i.e. any neighborhood of $x$ contains an infinite subset of $A$.). Since $F$ is closed, $x \in F \subset \mathscr{V}_{\gamma_{0}}^{*}$. Hence $x \in \bigcup_{n \in \omega} f_{\gamma_{0}}\left(x_{n}\right)$. This contradicts the fact that $x$ is an $\omega$-limit point of $A$.

COROLLARY 2.7. The following spaces are isocompact.

(1) neighborhood F-spaces. [13, Theorem 3.11]

(2) spaces satisfying property $\theta \mathbf{L}$. [6, Theorem 2.4]

(3) weakly $\left[\omega_{1}, \infty\right)^{r}$-refinable spaces. [21, Corollary 3.3]

(4) $\delta \theta$-penetrable spaces. [5, Theorem 3.B]

(5) pure spaces.[1, Theorem 5]

Proof. Apply Proposition 2.3 and Theorem 2.6.

Though an $\mathscr{F}$-space is isocompact [13, Theorem 3.11], the author does not know whether an $\mathscr{F}$-space is neat. The method of the proof of Theorem 2.6 leads to the following theorem, whose proof is omitted. A space is said to be $\omega_{1}$-compact if the cardinality of every closed discrete subset is countable. 
THEOREM 2.8. An $\omega_{1}$-compact $\omega_{1}$-neat $T_{1}$-space is a-realcompact.

COROLLARY 2.9. The following spaces are a-realcompact if they are $\omega_{1}$-compact $T_{1}$-spaces.

(1) spaces satisfying property $\theta \omega_{1} L .[6$, Theorem 2.5]

(2) weakly $\left[\omega_{1}, \infty\right)^{r}$-refinable spaces. [21, Corollary 3.6]

(3) $\delta \theta$-penetrable spaces. [4, Corollary 2.5]

(4) pure spaces.

Proof. Apply Proposition 2.3 and Theorem 2.8.

3. Mapping theorems and an example. The following lemma is easy.

LEMMA 3.1. Let $f$ be a closed map from $X$ onto $Y$ with Lindelöf fibers and $\mathscr{F}$ be a free closed ultrafilter on $X$ with c.i.p. Then $\mathscr{H}=\{H: H$ is closed in $Y$ and $\left.f^{-1} H \in \mathscr{F}\right\}$ is a free closed ultrafilter on $Y$ with c.i.p. such that $\lambda(\mathscr{F})=\lambda(\mathscr{H})$.

THEOREM 3.2. Let $f$ be a closed map from $X$ onto a $k$-neat space $Y$. If each fiber of $f$ is Lindelöf, then $X$ is $k$-neat.

Proof. Let $\mathscr{F}$ be a free closed ultrafilter on $X$ with c.i.p. Then by the above lemma $\mathscr{H}=\left\{H: H\right.$ is closed in $Y$ and $\left.f^{-1} H \in \mathscr{F}\right\}$ is a free closed ultrafilter on $Y$ with c.i.p. such that $\lambda(\mathscr{F})=\lambda(\mathscr{H})$. We get a $k$-neat system $\left\langle\left\{Y_{\gamma}\right\},\left\{\mathscr{V}_{\gamma}\right\},\left\{g_{\gamma}\right\}\right\rangle_{\gamma \in \Gamma}$ for $\mathscr{H}$. Put $X_{\gamma}=f^{-1} Y_{\gamma}, \mathscr{W}_{\gamma}=\left\{W_{x}\right.$ : $\left.x \in X_{\gamma}\right\}$, where $W_{x}=f^{-1}\left(g_{\gamma}(f(x))\right)$, and define a function $h_{\gamma}$ from $X_{\gamma}$ to $\mathscr{W}_{\gamma}$ such that $h_{\gamma}(x)=W_{x}$ for each $\gamma \in \Gamma$. It is easily seen that the system $\left\langle\left\{X_{\gamma}\right\},\left\{\mathscr{W}_{\gamma}\right\},\left\{h_{\gamma}\right\}\right\rangle_{\gamma \in \Gamma}$ is a desired $k$-neat system for $\mathscr{F}$.

COROLlaRY 3.3. A perfect preimage of a $k$-neat space is $k$-neat.

LEMMA 3.4. [14] Let $\mathscr{H}$ be a free closed ultrafilter on $X$ with c.i.p. If $B$ is a Borel set of $X$, and if $B$ contains no member of $\mathscr{H}$, then there exists $H \in \mathscr{H}$ such that $H \cap B=\varnothing$.

A space is said to be Borel complete [12], if every Borel ultrafilter on $X$ with c.i.p. is fixed. 
THEOREM 3.5. Let $f$ be a map from $X$ onto a Borel complete $T_{1}$-space $Y$. If each fiber of $f$ is $k$-neat, then $X$ is $k$-neat.

Proof. Let $\mathscr{H}$ be a free closed ultrafilter on $X$ with c.i.p. Set $\mathscr{B}=\left\{B: B\right.$ is a Borel set of $Y$ such that $f^{-1} B \supset H$ for some $\left.H \in \mathscr{H}\right\}$. It follows from Lemma 3.4 that $\mathscr{B}$ is a Borel ultrafilter on $Y$ with c.i.p. So $\cap \mathscr{B}=\{y\}$ for some $y \in Y$ (i.e. $f^{-1} y \in \mathscr{H}$ ). Put $E=f^{-1} y$ and $\mathscr{H} \mid E=$ $\{E \cap H: H \in \mathscr{H}\}$. We can easily see that $\mathscr{H} \mid E$ is a free closed ultrafilter on $E$ with c.i.p. such that $\lambda(\mathscr{H})=\lambda(\mathscr{H} \mid E)$. Let $\left\langle\left\{E_{\gamma}\right\},\left\{\mathscr{V}_{\gamma}\right\},\left\{g_{\gamma}\right\}\right\rangle_{\gamma \in \Gamma}$ be a $k$-neat system for $\mathscr{H} \mid E$. We extend this system in the following manner. Set $\mathscr{W}_{\gamma}=\left\{V \cup X-E: V \in \mathscr{V}_{\gamma}\right\}$ and define a function $h_{\gamma}$ from $E_{\gamma}$ to $\mathscr{W}_{\gamma}$ such that $h_{\gamma}(x)=g_{\gamma}(x) \cup X-E$ for each $\gamma \in \Gamma$. We get a system consisting of $\left\{E_{\gamma}, X-E: \gamma \in \Gamma\right\},\left\{\mathscr{W}_{\gamma},\{X-E\}: \gamma \in \Gamma\right\}$ and $\left\{h_{\gamma}, j: \gamma \in \Gamma\right\}$, where $j$ is the trivial function from $X-E$ to $\{X-E\}$. This system is a desired one for $\mathscr{H}$.

Corollary 3.6. A product of a Borel complete $T_{1}$-space and a $k$-neat space is $k$-neat.

The same method of the proof of Theorem 3.5 leads to the following theorem, whose proof is omitted.

THEOREM 3.7. Let $f$ be a closed map from $X$ onto an a-realcompact $T_{1}$-space. If each fiber of $f$ is $k$-neat, then $X$ is $k$-neat.

EXAMPLE 3.8. We give a neat space that is not an $\mathscr{F}$-space, not a pure space, not a weakly $\left[\omega_{1}, \infty\right)^{r}$-refinable space and not satisfying property $\theta L$. Let $X$ be an hereditarily separable non-Lindelöf space constructed in [16] under the continuum hypothesis. $X$ is a $\theta$-penetrable space (hence a neat space) that is not weakly $\delta \theta$-refinable [4, Remarks 2.1.(b)]. $X$ is not even weakly $\left[\omega_{1}, \infty\right)^{r}$-refinable because the cardinality of $X$ is $\omega_{1}$. Moreover $X$ does not satisfy property $\theta L$ by [6, Theorem 2.8], and $X$ is not an $\mathscr{F}$-space by [13, Theorem 3.3]. Let $Y$ be the Tychonoff space mentioned in $[11,9 \mathrm{~L}]$. Since $Y$ is a $P$-space (i.e. $G_{\delta}$-sets are open), it is neat. Since $Y$ is an $\omega_{1}$-compact non- $a$-realcompact space [4, Remarks 2.7], by Corollary 2.9 (4), $Y$ is not pure. By Corollary 3.6 $X \times Y$ is neat because $X$ is hereditarily realcompact (hence Borel complete [12, Theorem 3.6]). Obviously $X \times Y$ is not an $\mathscr{F}$-space, not a pure space, not a weakly $\left[\omega_{1}, \infty\right)^{r}$ refinable space and not satisfying property $\theta L$. 
REMARK 3.9. The above space $Y$ answers some questions in [6] and [8]. Since a $P$-space satisfies property $L, Y$ affirmatively answers Question $3.3,3.4$ and 3.5 in [6], because $Y$ is an $\omega_{1}$-compact non- $a$-realcompact $P$-space (as mentioned in Example 3.8 ) which is not weakly $\delta \theta$-refinable by [3, Corollary 3.3]. Question 3.3 in [6] was already answered in [8, Example 2.2], but the space is not regular though it is $T_{2}$. The space $Y$ negatively answers Question 4.2 in [8]. Because an $\omega_{1}$-compact $P$-space must be preparacompact.

4. Isocompact spaces having a countably compact dense subset. In this section we shall consider the following question. When are isocompact spaces having a countably compact dense subset compact? For this question we can obtain some answers from already known results. For example the next proposition holds.

LEMMA 4.1. Let $\mathscr{U}$ be an open cover of a countably compact space $X$. If there exists an interlacing $\mathscr{E}=\bigcup_{n \in \omega} \mathscr{E}_{n}$ on $X$ such that, for each $n \in \omega$ and $x \in \mathscr{E}_{n}^{*}, \operatorname{St}\left(x, \mathscr{E}_{n}\right) \subset \mathscr{V}^{*}$ for some countable subfamily $\mathscr{V} \subset \mathscr{U}$, then $\mathscr{U}$ has a finite subcover of $X$.

Proof. This lemma is proved the same way as [19, Theorem 1.1].

Proposition 4.2. The following spaces are compact if they are regular $T_{2}$-spaces having a countably compact dense subset.

(1) weakly Borel complete spaces.

(2) spaces satisfying property $\theta L$.

(3) $\delta \theta$-penetrable spaces.

(4) ultrapure spaces.

Proof. (1) is due to [18, Theorem 2.1]. We prove the case of (2). Let $\mathscr{U}$ be any open cover of $X$ and $\mathscr{V}$ be an open cover of $X$ such that for each $V \in \mathscr{V}$ there exists $U \in \mathscr{U}$ such that $\bar{V} \subset U$. For this $\mathscr{V}$, since $X$ satisfies property $\theta L$, we can take sequences $\left\langle\mathscr{D}_{n}: n \in \omega\right\rangle$ and $\left\langle\mathscr{V}_{n}: n \in \omega\right\rangle$ in Definition 1.3. Let $Y$ be a countably compact dense subset of $X$. If we restrict the discussion of [6, Theorem 2.4] to $Y$, we can obtain a countable subfamily $\mathscr{W}$ of $\mathscr{V}$ which covers $Y$. So $Y$ is covered by a finite subfamily of $\mathscr{W}$. Since $Y$ is dense in $X$, we can take a finite subcover of $X$ from $\mathscr{U}$. (3) and (4) are similarly proved by [5, Theorem 3.B] and Lemma 4.1 respectively.

A space is said to be CL-isocompact [18] if the closure of every countably compact subset is compact. Since each property of Proposition 4.2 is closed hereditary, we get the following corollary. 
COROLLARY 4.3. The following spaces are CL-isocompact if they are regular $T_{2}$.

(1) weakly Borel complete spaces.

(2) spaces satisfying property $\theta L$.

(3) $\delta \theta$-penetrable spaces.

(4) ultrapure spaces.

For a general case we shall prove the next theorem.

THEOREM 4.4. Let $X$ be a regular $T_{2}$ isocompact space. If $X$ is represented as the union of a countably compact dense subset $X_{1}$ and an almost realcompact dense subset $X_{2}$, then $X$ is compact.

Proof. Firstly we show that $X$ is almost realcompact. Let $\mathscr{U}$ be an open ultrafilter on $X$, and suppose that $\overline{\mathscr{U}}$ has c.i.p. Put $\mathscr{U} \mid X_{2}=\left\{U \cap X_{2}\right.$ : $\underline{U \in \mathscr{U}}\}$. Then it is easily seen that $\mathscr{U} \mid X_{2}$ is an open ultrafilter on $X_{2}$. If $\overline{\mathscr{U} \mid X_{2}^{X_{2}}}$ has c.i.p., then $\cap \overline{\mathscr{U} \mid} \mid X_{2}^{X_{2}} \neq \varnothing$ by almost realcompactness of $X_{2}$. Hence $\cap \overline{\mathscr{U}} \neq \varnothing$. If $\overline{\mathscr{U} \mid X_{2}^{X_{2}}}$ has not c.i.p., then there exists a countable subfamily $\mathscr{V} \subset \mathscr{U}$ such that $(\cap \overline{\mathscr{V}}) \cap X_{2}=\varnothing$. Since $\cap \overline{\mathscr{V}}$ is countably compact closed in $X$, it is compact. So we get $\cap \overline{\mathscr{U}} \neq \varnothing$. Thus $X$ is almost realcompact. Now we consider the absolute $E X$ of $X$ [20]. Since $E X$ is realcompact [20, Theorem 4.6] and pseudocompact, it is compact. We conclude that $X$ is compact.

COROLlaRY 4.5. Let $X$ be a regular $T_{2}$ neat space. If $X$ is represented as the union of a countably compact dense subset and an almost realcompact dense subset, then $X$ is compact.

EXAMPLE 4.6. We cannot omit the regularity of Theorem 4.4. Let $X$ be Tychonoff plank [11] (i.e. $\left.X=\omega_{1}+1 \times \omega+1-\left\{\left(\omega_{1}, \omega\right)\right\}\right)$ and $Y$ be the space obtained from $X$ by contracting $\omega_{1} \times\{\omega\}$ to the one point. Though this $T_{2}$-space $Y$ satisfies all conditions of Theorem 4.4 except the regularity, $Y$ is not compact.

\section{REFERENCES}

[1] A. V. Arhangel'skii, The star method, new classes of spaces and countably compactness, Soviet Math. Dokl., 21, no. 2 (1980), 550-554.

[2] P. Bacon, The compactness of countably compact spaces, Pacific J. Math., 32 (1970), 587-592.

[3] R. L. Blair, Closed-completeness in spaces with weak covering properties, Set Theoretic Topology, Academic Press, (1977), 17-45. , On a theorem of Chaber, Topology Proc., 5 (1980), 33-46. 
[5] J. Chaber, Conditions which imply compactness in countably compact spaces, Bull. Acad. Polon. Sci. Ser. Math., 24 (1976), 993-998.

[6] S. W. Davis, A cushioning-type weak covering property, Pacific J. Math., 80 (1979), 359-370.

[7] _ On $\mathscr{F}_{r}$-spaces, General Topology and Appl., 9 (1978), 131-138.

[8] S. W. Davis and J. C. Smith, The paracompactness of preparacompact spaces, Topology Proc., 4 (1979), 345-360.

[9] N. Dykes, Generalizations of realcompact spaces, Pacific J. Math., 33 (1970), 571-581.

[10] Z. Frolík, A generalization of realcompact spaces, Czech. Math. J., 13 (1963), 127-138.

[11] L. Gillman and M. Jerison, Rings of continuous functions, Princeton (1960).

[12] A. W. Hager, G. D. Reynolds and M. D. Rice, Borel-complete topological spaces, Fund. Math., 75 (1972), 135-143.

[13] P. W. Harley, III and R. M. Stephenson, Jr., Symmetrizable and related spaces, Trans. Amer. Math. Soc., 219 (1976), 89-111.

[14] A. Hayes, Alexander's theorem for realcompactness, Proc. Cambridge Phil. Soc., 64 (1968), 41-43.

[15] R. E. Hodel, Metrizability of topological spaces, Pacific J. Math., 55 (1974), 441-449.

[16] I. Juhász, K. Kunen and M. E. Rudin, Two more hereditarily separable non-Lindelöf spaces, Canad. J. Math., 28 (1976), 998-1005.

[17] M. D. Rice and G. D. Reynolds, Weakly Borel-complete topological spaces, Fund. Math., CV (1980), 179-185.

[18] M. Sakai, CL-isocompactness and weak Borel completeness, Tsukuba J. Math., 8 (1984), 377-382.

[19] H. H. Wicke and J. M. Worrell, Jr., Point-countability and compactness, Proc. Amer. Math. Soc., 55 (1976), 427-431.

[29] R. G. Woods, A survey of absolutes of topological spaces, Topological Structures II, Math. Centre Tracts, 116 (1979), 323-362.

[21] J. M. Worrell, Jr. and H. H. Wicke, A covering property which implies isocompactness, I, Proc. Amer. Math. Soc., 79 (1980), 331-334.

Received April 14, 1984 and in revised form June 1, 1985.

UNIVERSITY OF TSUKUBA

IBARAKI, 305 JAPAN 



\section{PACIFIC JOURNAL OF MATHEMATICS EDITORS}

\author{
V. S. VARADARAJAN (Managing Editor) \\ University of California \\ Los Angeles, CA 90024 \\ Hebert Clemens \\ University of Utah \\ Salt Lake City, UT 84112 \\ Charles R. DePrima \\ California Institute of Technology \\ Pasadena, CA 91125
}

R. FINN

Stanford University

Stanford, CA 94305

HermanN FlaschKa

University of Arizona

Tucson, AZ 85721

RAMESH A. GANGOLlI

University of Washington

Seattle, WA 98195

ROBION KIRBY

University of California

Berkeley, CA 94720

ASSOCIATE EDITORS
R. ARENS
E. F. BECKENBACH
B. H. NEUMANN
F. WOLF
K. YoshidA (1906-1982)

C. C. Moore

University of California

Berkeley, CA 94720

H. SAMELSON

Stanford University

Stanford, CA 94305

HAROLD STARK

University of California, San Diego

La Jolla, CA 92093

\section{SUPPORTING INSTITUTIONS}

UNIVERSITY OF ARIZONA

UNIVERSITY OF BRITISH COLUMBIA

CALIFORNIA INSTITUTE OF TECHNOLOGY

UNIVERSITY OF CALIFORNIA

MONTANA STATE UNIVERSITY

UNIVERSITY OF NEVADA, RENO

NEW MEXICO STATE UNIVERSITY

OREGON STATE UNIVERSITY
UNIVERSITY OF OREGON

UNIVERSITY OF SOUTHERN CALIFORNIA

STANFORD UNIVERSITY

UNIVERSITY OF HAWAII

UNIVERSITY OF TOKYO

UNIVERSITY OF UTAH

WASHINGTON STATE UNIVERSITY

UNIVERSITY OF WASHINGTON 


\section{Pacific Journal of Mathematics}

Vol. 122, No. $1 \quad$ January, 1986

Michael James Cambern, Near isometries of Bochner $L^{1}$ and $L^{\infty}$ spaces ....1 Kun Soo Chang, Gerald William Johnson and David Lee Skoug, The

Feynman integral of quadratic potentials depending on two time

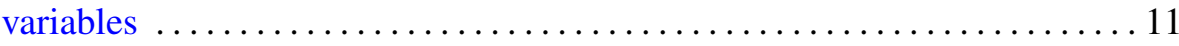

Robert Coleman, One-dimensional algebraic formal groups $\ldots \ldots \ldots \ldots \ldots 35$

Alberto Collino, The Abel-Jacobi isomorphism for the cubic fivefold .......43

N. J. Dev and S. S. Khare, Finite group action and vanishing of $N_{*}^{G}[F] \ldots 57$

Harold George Diamond and Jeffrey D. Vaaler, Estimates for partial sums

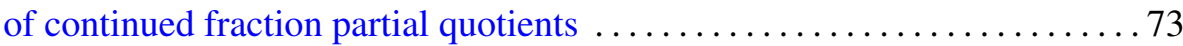

Kenneth R. Goodearl, Patch-continuity of normalized ranks of modules

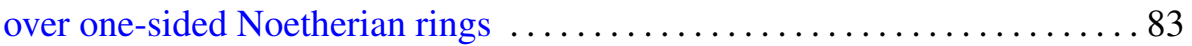

Dean Robert Hickerson and Sherman K. Stein, Abelian groups and

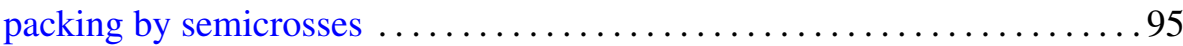

Karsten Johnsen and Harmut Laue, Fitting structures $\ldots \ldots \ldots \ldots \ldots 11$

Darren Long, Discs in compression bodies . ................... 129

Joseph B. Miles, On the growth of meromorphic functions with radially distributed zeros and poles ........................... 147

Walter Volodymyr Petryshyn, Solvability of various boundary value problems for the equation $x^{\prime \prime}=f\left(t, x, x^{\prime}, x^{\prime \prime}\right)-y \ldots \ldots \ldots \ldots \ldots . \ldots 169$

Elżbieta Pol, The Baire-category method in some compact extension problems

Masami Sakai, A new class of isocompact spaces and related results 211

Thomas Richard Shemanske, Representations of ternary quadratic forms and the class number of imaginary quadratic fields ..

Tsuyoshi Uehara, On class numbers of cyclic quartic fields 